\title{
ANOMALOUS REDSHIFTS IN GALAXIES AND QUASARS
}

\author{
Halton Arp \\ Hale Observatories, Carnegie Institution of Washington, California \\ Institute of Technology, Pasadena, California, U.S.A.
}

\begin{abstract}
Les associations apparentes entre galaxies et quasars sont récapitulées. Les probabilités d'associations accidentelles sont discutées et les relations entre la séparation des quasars et la nature de la galaxie associée sont présentées. De nouveaux cas d'associations apparentes entre galaxies de faible décalage vers le rouge et de grand décalage sont présentés et discutés. Le phénomène des décalages systématiquement plus grands pour les galaxies non elliptiques dans les amas et les groupes de galaxies est abordé. Enfin de récentes évidences sur les galaxies compagnons de $M 31$ et $M 81$ montrent que parmi les douze compagnons certains, tous ont un décalage spectral plus grand que la galaxie principale.
\end{abstract}

\section{INTRODUCTION}

In the three years since the last I. A.U., further evidence for the existence of anomalous (non-Doppler) redshifts has accumulated. The present review will start from the status as reported in I. A.U. Symposium 58 held in Canberra in 1973 (see Arp 1974a). First, new evidence for association of quasars with low redshift galaxies will be discussed and then new evidence for association of high redshift galaxies with low redshift galaxies. Some astronomers feel that the two subjects are somewhat separate since the quasars are so unusual that they might involve unknown redshift mechanisms whereas it would be much more difficult to invoke such mechanisms for galaxies of indubitable gaseous and stellar content. On the other hand, most astronomers believe that there is some kind of continuity of physical characteristics between quasars and compact, active galaxies and this author further believes that the intrinsic redshifts of the quasars are of the same 
nature as the lesser components of intrinsic redshifts observed in galaxies.

Therefore, the following review will discuss the evidence for anomalous redshifts in both quasars and galaxies. The aim will be first to establish as unequivocally as possible from the accumulation of evidence that non-Doppler redshifts do exist. This will be done by reference to both statistical probabilities of associations as well as interactions between different redshift objects. A secondary aim of this review will be to elicit observational correlations between these excess redshifts and other physical parameters in order to give some basis for trying to arrive at a theoretical understanding of the redshift phenomenon.

Table 1 does not contain all currently known associations of galaxies and quasars. For example, it does not mention the quasars 0823+033 and 1021-00 (Browne and McEwan 1973), the quasar 1953-325 (Bolton and Peterson, see Arp 1974a) a quasar near $01^{\mathrm{h}} 22^{\mathrm{m}}$ and $+10^{\circ} 50^{\prime}$ (Hazard, Mitten and Murdoch, private communication) and 0937-011 and 1302-206 (Bolton and Peterson, private communication). The Table does contain, however, the better known associations for which a moderate amount of information is available. The entries above the double line are post-1973 and show that the number of associations has more than doubled in the last three years. These latest additions are particularly impressive because nine of them are radio-quiet quasars which have not been looked for systematically around galaxies but have just been discovered accidentally or on inspection of particular galaxies.

\section{NEW QUASAR-GALAXY ASSOCIATIONS}

The probability that these apparent associations could be caused by background quasars accidentally being projected near foreground galaxies needs first to be estimated. We see by referring to Table 3 of Arp, Baldwin and Wampler (1975) that, assuming the conventional estimate of background density of radioquiet quasars, the probability of getting a quasar of the apparent magnitudes listed in the first part of Table 1 falling that close to any given point in the sky is of the order of magnitude of one in a thousand. The surroundings of very few galaxies have been searched for radio-quiet quasars - considerably less than 100 galaxies. To find the number of examples listed in Table 1 of the present paper clearly demands that some quasars are preferentially associated with galaxies. In the case of quasars next to companion galaxies, the only possible counterargument to this conclusion would be to argue that radio-quiet quasars were several orders of magnitude more frequent in the field than they are currently believed to be. Such check investigations are in progress, but it is my opinion that the burden of proof presently falls on those who contend the associations are only accidental.

Compelling as these probabilities may be, however, there is considerable additional evidence in favor of the reality of these associations due to the special character of many of the configurations.

(a) Galaxy-Companion-Quasar Associations 


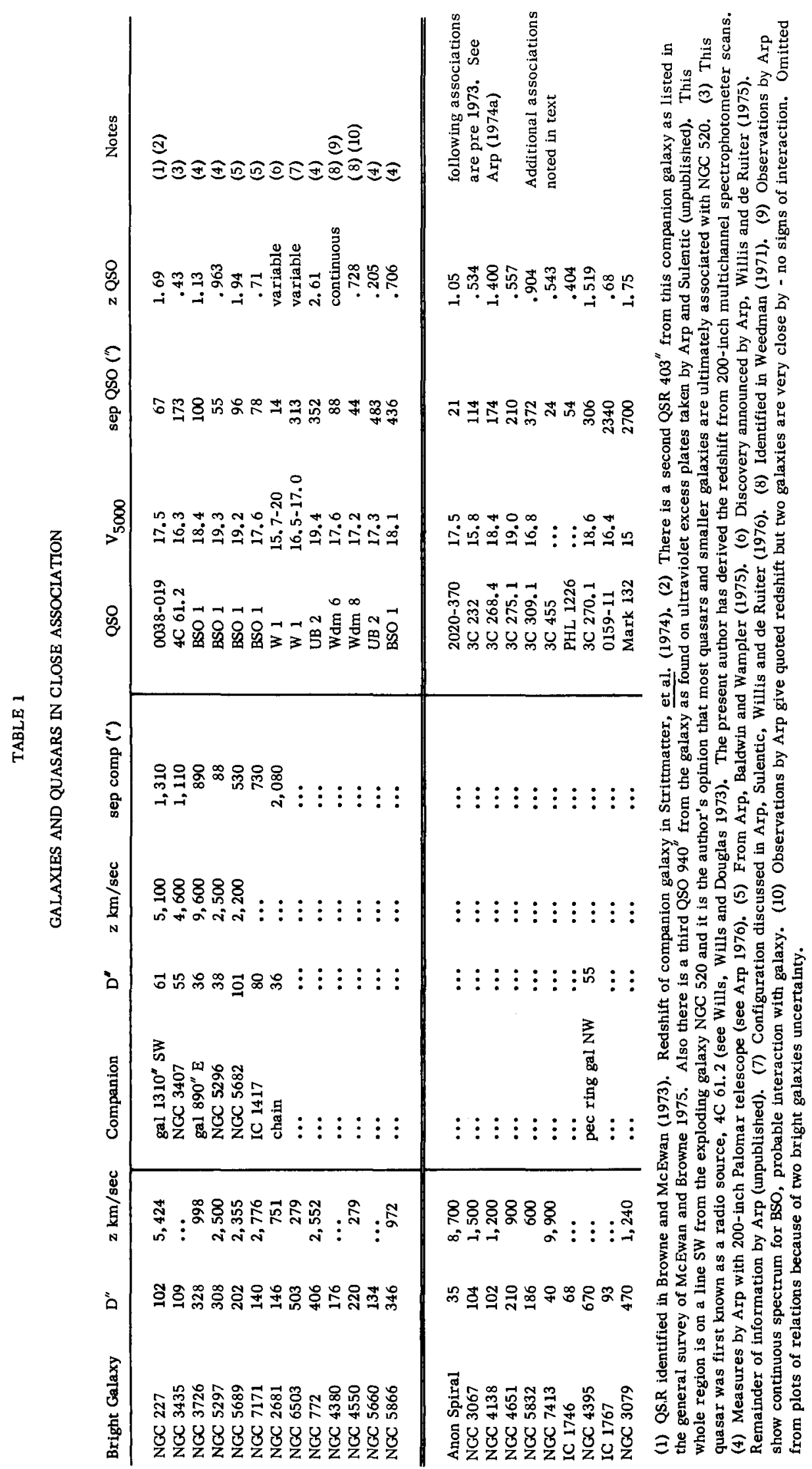


Arp (1972b) advanced the hypothesis that smaller companion galaxies in the vicinity of large galaxies were more often in early stages of evolution characterized by secondary ejection, fission, and disruption. Therefore, it was expected that if quasars were ejected from galaxies they would be more numerous around these companion galaxies. An exploratory nonsystematic search for such configurations was made, and the first two cases investigated showed two new quasars very near companion galaxies (Arp, Baldwin, and Wampler 1975). The properties of these first two configurations are listed in Table 1 across from the large central galaxies which are at the center of each configuration, NGC 5689 and NGC 7171.

Subsequently, and again in casual searches, four more examples of companion galaxies with closely associated quasars were discovered. They are also listed in Table 1 across from the central galaxies NGC 227, NGC 3435, NGC 3726, and NGC 5297. Some additional data on these last three are in press (Arp 1976). It should be commented that in the case of the quasars next to NGC 227 and NGC 3407, there were radio sources already known to be quasars (Browne and McEwan 1973; Wills, Wills, and Douglas 1973). The present author has determined only the redshift of 4C61.2. In the case of NGC 5296 and the companion to NGC 3726, however, the author has looked for the blue stellar object near the companion and then demonstrated it to be a quasar with the redshift reported in Table 1.

From the standpoint of probability of association, we are not dealing with NGC galaxies in general now. We are specifying just bright galaxies, fairly isolated with obvious companion galaxies. Therefore, the pool from which these candidate configurations are drawn is much restricted. Yet we are having a very high rate of success - I would estimate better than 50\%. The precise criteria of galaxycompanion galaxy have now been discovered and so systematic searches can be made. But, pending the completion of such searches, we can cite independent physical evidence for the association of some of these galaxies with quasars.

\section{(b) Evidence for Galaxy-Quasar Interaction}

In some associations there is very strong evidence for physical interaction of the quasar with material in the galaxy. The best example of this is shown in photograph of NGC 5297 and 5296 shown in Figure 1. Only after the identification of the quasar had been confirmed did deep exposures reveal the luminous extension from the companion galaxy NGC 5296 pointing to the quasar. It also turned out that the compact, peculiar galaxy just south of NGC 5296 has a narrow absorption lane just outside its northern perimeter which is silhouetted against the background of NGC 5296. Since this small galaxy has a redshift of $z=25,900 \mathrm{~km} / \mathrm{sec}$ and has to be in front of NGC 5296 which has a redshift of $z=2,500 \mathrm{~km} / \mathrm{sec}$, it is a strong example of a galaxy with an anomalously high redshift.

Another good example of interaction is that of NGC 5682-quasar, where there is a separate luminous filament, beside the two spiral arms in the disturbed galaxy which points just in the direction of the quasar. Galáxies nearest the quasars 


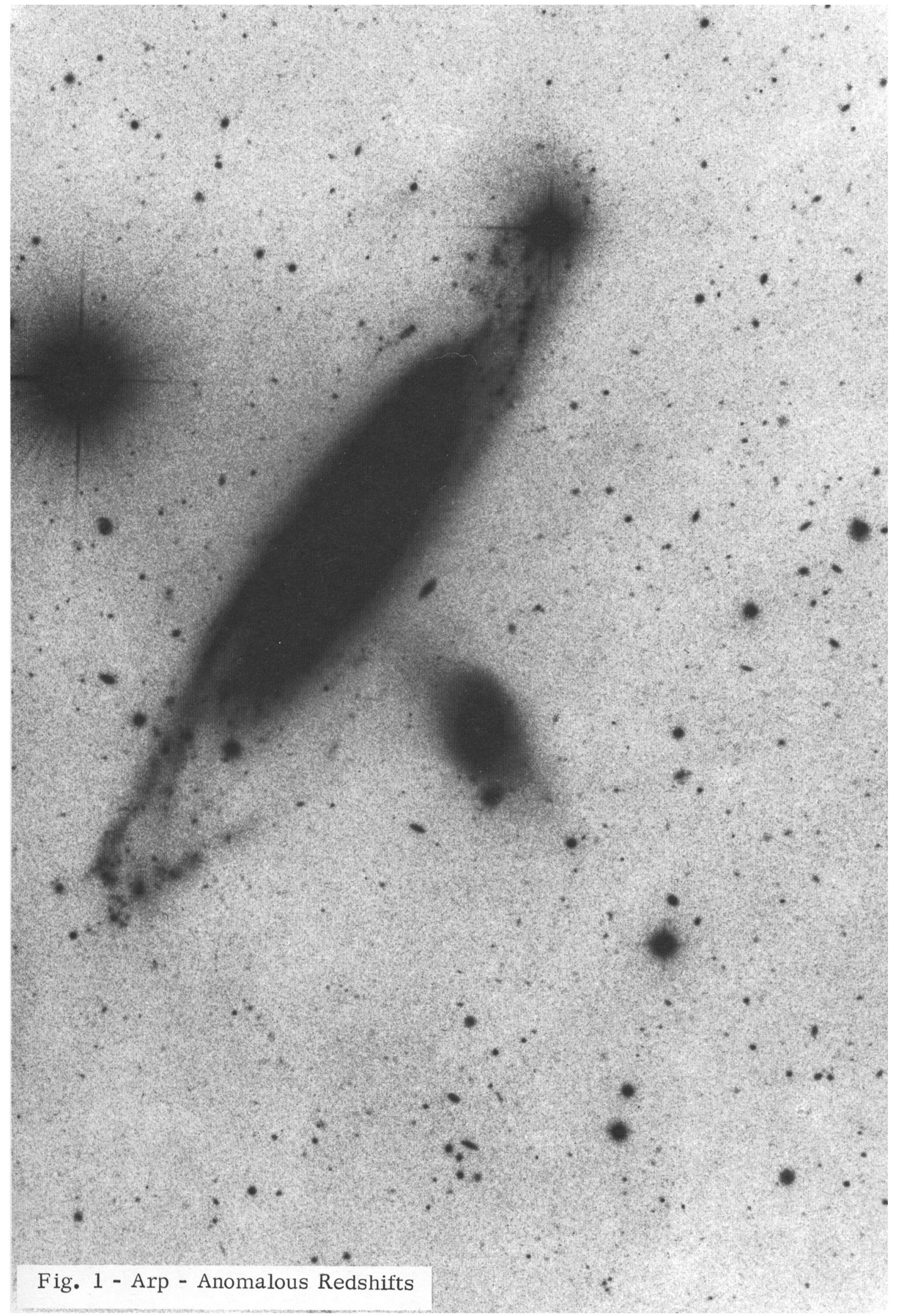


0038-019, NGC $2681 \mathrm{~W} 1$, NGC $6503 \mathrm{~W} 1$, NGC 772, Weedman \#6, NGC 5600 UB2, NGC 5866 BSO 1, 2020-370, 3C 232, 3C 455, PHL 1226, 3C 270.1, 0159-11, and Mark 132 are all disturbed in ways which could be logically attributed to the presence, or recent presence, of the quasar.

In 1975 Arp, Pratt, and Sulentic showed that there was strong evidence for interaction in all four cases where quasars were projected nearest to galaxies $(\leq 55$ arc sec). From the updated list in Table 1 we can now report that out of seven new associations where the quasar is $<100$ arc sec from the nearest galaxy, five cases show moderate to strong evidence for interaction with the galaxy. In total we have nine out of the 11 closest projections showing evidence of interaction, and four cases (NGC 5296, NGC 5682, NGC 4319, and NGC 7413) showing, in this author's opinion, exceedingly strong evidence for interaction.

\section{(c) Correlation of Properties of Associated Galaxies and Quasars}

If the quasars in Table 1 are actually associated with the galaxies, it is natural to ask whether those associated with more nearby galaxies appear more widely separated and those associated with more distant galaxies are at smaller apparent separations. Burbidge, O'Dell, and Strittmatter (1972) investigated this question by plotting log of the redshift of the associated galaxies against log of the separation of the galaxy-quasar for the five associations then known. Their points are shown as filled circles in Figure 2. Most of these new points fall close to the original Burbidge, O'Dell, and Strittmatter line. The Weedman object close to NGC 4550 is here believed to be unassociated or greatly projected because there is no sign of interaction with the galaxy. But its presence does prove quasars can be discovered very close to large galaxies, and that this obvious lower limit for quasar separations in Figure 2 is not the result of a selection effect whereby the galaxy blocks discovery of quasars within its diameter.

As explained in the caption of Figure 2, the solid line represents what the quasar separations should be if they were all orbiting around galaxies at exactly $20 \mathrm{kpc}$ distance and were seen at maximum elongation. The dashed line below represents a reduction by $\sqrt{2}$ for average projection factors. Of course, there is no reason why the quasars should be in bound orbits. They could be passing outward from the galaxy and, at this distance, simply in a stage of their development particularly favorable for their discovery. But regardless of the physical nature of the association, characteristic separations should, on the average, be wider for nearby galaxies and smaller for more distant galaxies. The importance of Figure 2 is that it shows this expectation is fulfilled. In contrast, if the quasars were background objects, not associated with the galaxies, the points should scatter in an uncorrelated fashion across the plane of Figure 2. 


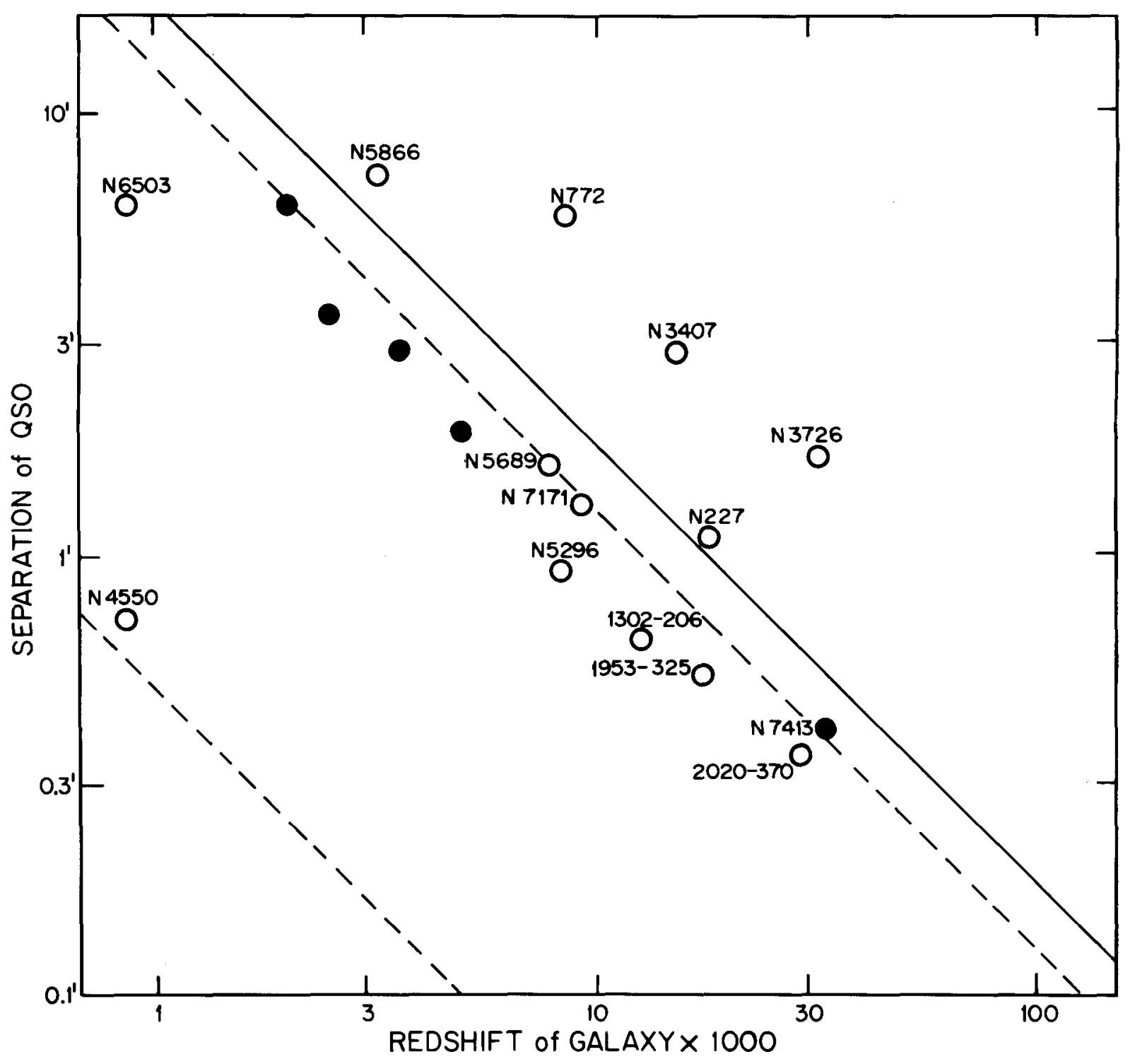

Figure 2. Apparent separation of quasars from galaxies plotted against redshift of galaxy. Solid line represents quasars at $20 \mathrm{kpc}$ distance from galaxy all seen at maximum elongation, dashed line at an average projection reduced by $\sqrt{2}$. Filled circles are original 5 cases treated by Burbidge, O'Dell and Strittmatter (1972) and open circles are new cases.

Another way of demonstrating the correlation of galaxy-quasar separation with galaxy distance is shown in Figure 3, where the diameter of the nearest galaxy to the quasar - a measure of distance to the galaxy - is plotted versus apparent galaxy-quasar separation. Again, the older associations are plotted as filled circles and the newer ones from the first part of Table 1 are plotted as open circles. There are more points in this diagram than in Figure 2 because the apparent diameter-distance criterion was available for some galaxies that did not have the redshift-distance criterion available. Again, we see the rough correlation that quasars appear close by to small galaxies and further away from larger galaxies, 
as they would if the large galaxies were on the average nearer and the smaller galaxies on the average more distant.

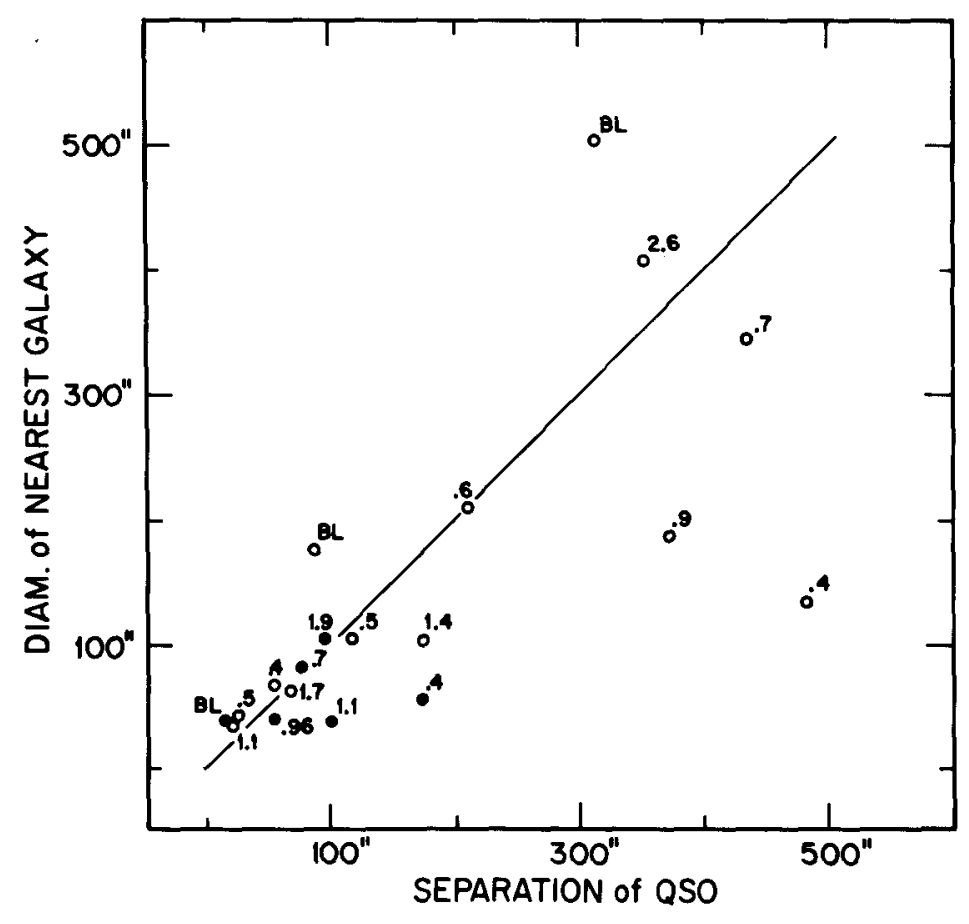

Figure 3. Apparent separation of quasars from galaxies plotted against apparent diameter of nearest galaxy. Filled circles are pre-1973 cases, open circles are newer cases from Table 1. Redshifts of quasars or BL Lac continuum type character is noted beside each quasar point. A general correlation between apparent size of galaxy and apparent separation of quasar is seen but BL Lac and high $z$ quasars appear closest, and lowest redshift quasars farthest, from the galaxies.

But a very interesting additional trend is apparent from Figure 3. All three of the points falling furthest toward the small separation side of the relation are BL Lac (continuum)-type quasars. In contrast, the lowest redshift quasars of $z$ $=0.4$ fall to the largest separation side of the relation. This suggests to the author that, as the quasars are ejected outward from the galaxies and as time passes, they first become visible as continuum, variable BL Lac-type objects, then evolve evolve into high-redshift quasars, and finally into (low-luminosity) low-redshift quasars.

(d) Absolute Magnitudes of Associated Quasars

If we assume for working purposes that the redshifts of the associated galaxies are generally indicative of distance to the association, we may calculate distance 
moduli for a number of the quasars listed in Table 1. Using a Hubble constant of $H=80 \mathrm{~km} / \mathrm{sec} / \mathrm{Mpc}$, thirteen quasars can be plotted in the luminosity-redshift diagram of Figure 4. Judged by its large diameter, NGC 772 is probably closer than its redshift would indicate, NGC 227 is probably at the closer distance of NGC 520, and the chain involved with 2020-370 may also be overestimated in distance from its redshift. So the three highest points in Figure 4 are the least trustworthy, and we therefore conclude that the actual luminosities of the quasars are best represented by the ten points in the lower left of the diagram. As

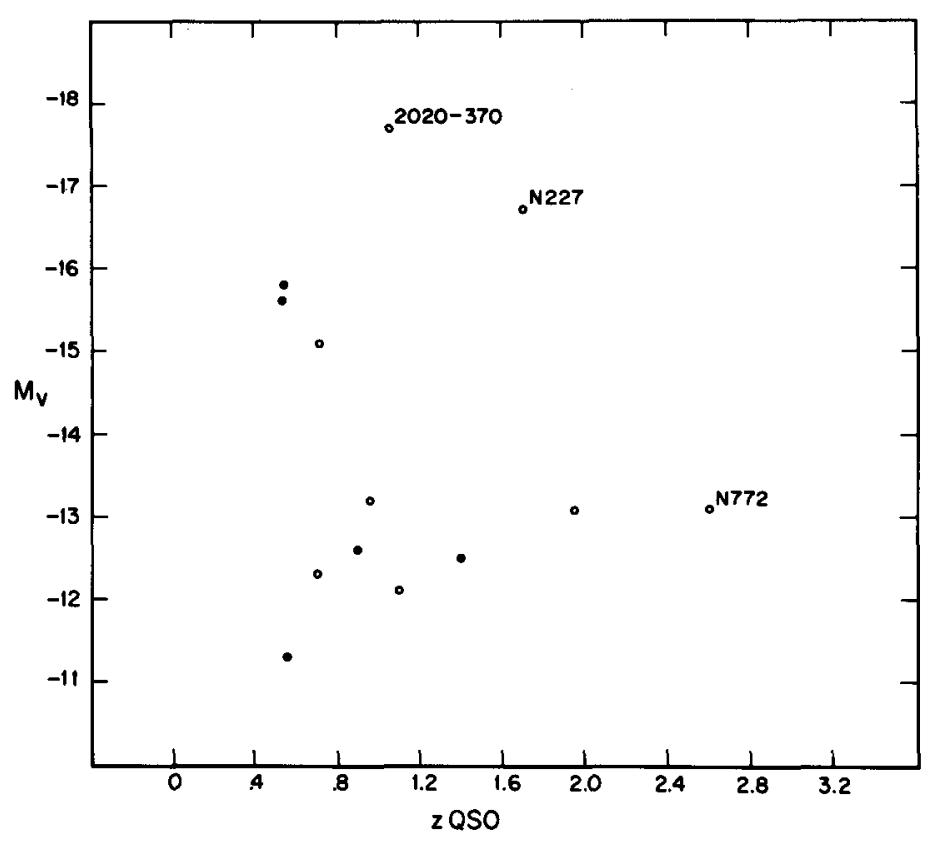

Figure 4. Quasar absolute magnitudes as a function of their redshift. Closed and open circles are old and new cases respectively. Distance moduli computed from redshifts of associated galaxies using a Hubble constant of $H=80 \mathrm{~km} / \mathrm{sec} / \mathrm{Mpc}$. Points marked NGC 772, NGC 227 and 2020-370 are most likely to have overestimated distances and therefore the average luminosity for quasars is probably best represented by the 10 cases in the lower left of the diagram.

concluded previously (Arp 1974b), the luminosities of the quasars are lowest near $z=0.4$ and $z=2$ and attain maximum brightness in between. Considering the well-known propensity for quasars to be violently variable in apparent magnitude, however, it is probably not to be expected that they would show a very precisely defined luminosity-intrinsic redshift relation.

\section{SPECIAL CASE OF RADIO SOURCES AND A QUASAR ON A LINE FROM AN ELLIPTICAL GALAXY}


In an important paper published in 1975, Ekers, Fanti, Lari, and M. H. Ulrich call attention to a Bologna radio source B20925+30. Two extended and three compact radio sources extend from either side of a central $E$ galaxy, so closely aligned that the authors calculate the accidental chance of this configuration to be only of the order of $10^{-5}$.

One of the compact radio sources was identified very accurately with a faint blue star. Arp (unpublished) reports in the present paper that observations with both the multichannel spectrophotometer and the SIT digital spectrograph on the 5-meter telescope at Palomar show that this star is indeed a quasar. The derived redshift is: $z$ (emission lines) $=2.02, z$ (absorption) $=1.95$.

The quasar has a visual apparent magnitude of between $\mathrm{m}_{\mathrm{v}}=21$ and $21.5 \mathrm{mag}$. As such, it may be the faintest quasar so far to have its redshift measured. The important point is, of course, not so much the exact value of its redshift, but that it is unquestionably identified as a quasar. The idea that elliptical galaxies eject radio sources has long been accepted. That some of these radio sources could be high-redshift quasars is an obvious extension of the ejection hypothesis (see, for example, the ejection process from the E galaxy, NGC 4486, as discussed by Arp 1972a).

The redshift of the central galaxy of $\mathrm{B} 20925+30$ is $z=0.0266$, and using $H=$ $80 \mathrm{~km} / \mathrm{sec} / \mathrm{Mpc}$ gives a distance of $100 \mathrm{Mpc}$. This means the quasar just discussed should have an absolute magnitude between $M_{V}=-13.5$ to $-14 \mathrm{mag}$. That fits well with the other points in Figure 4. At 8 ' from the central galaxy, the quasar is at an unusually large separation in terms of Figure 2. But most quasars are associated with spiral galaxies, and the few that are associated with elliptical galaxies may be ejected with greater speeds - as one possible suggestion.

It is also significant, I feel, that there are six other quasars within $11 / 2^{\circ}$ of B20924+30 (Fanti, et al. 1975).

\section{NEW DISCORDANT REDSHIFT GALAXIES}

Since the discordant redshifts in galaxy associations were last reviewed (Arp 1973), about seven new and very clear examples have become known. They are:

1. A compact, peculiar galaxy south of NGC 5296, which is shown in Figure 1 and discussed in Section II.

2. A very compact object (almost stellar with a narrow, bifurcating absorption lane) is located just on the southwest edge of NGC 1199. Figure 5 shows that this object obscures in a rim form the outskirts of the E galaxy NGC 1199, which must be behind it. The compact object has a very st rong emission-line spectrum at about $z=13,300 \mathrm{~km} / \mathrm{sec}$, whereas NGC 1199 has a redshift near $z=2,670 \mathrm{~km} / \mathrm{sec}$. Clearly, as in the preceding case involving NGC 5296, the higher redsh ift galaxy is in front of the lower redshift galaxy and, therefore, its redshift is much in excess of what would 


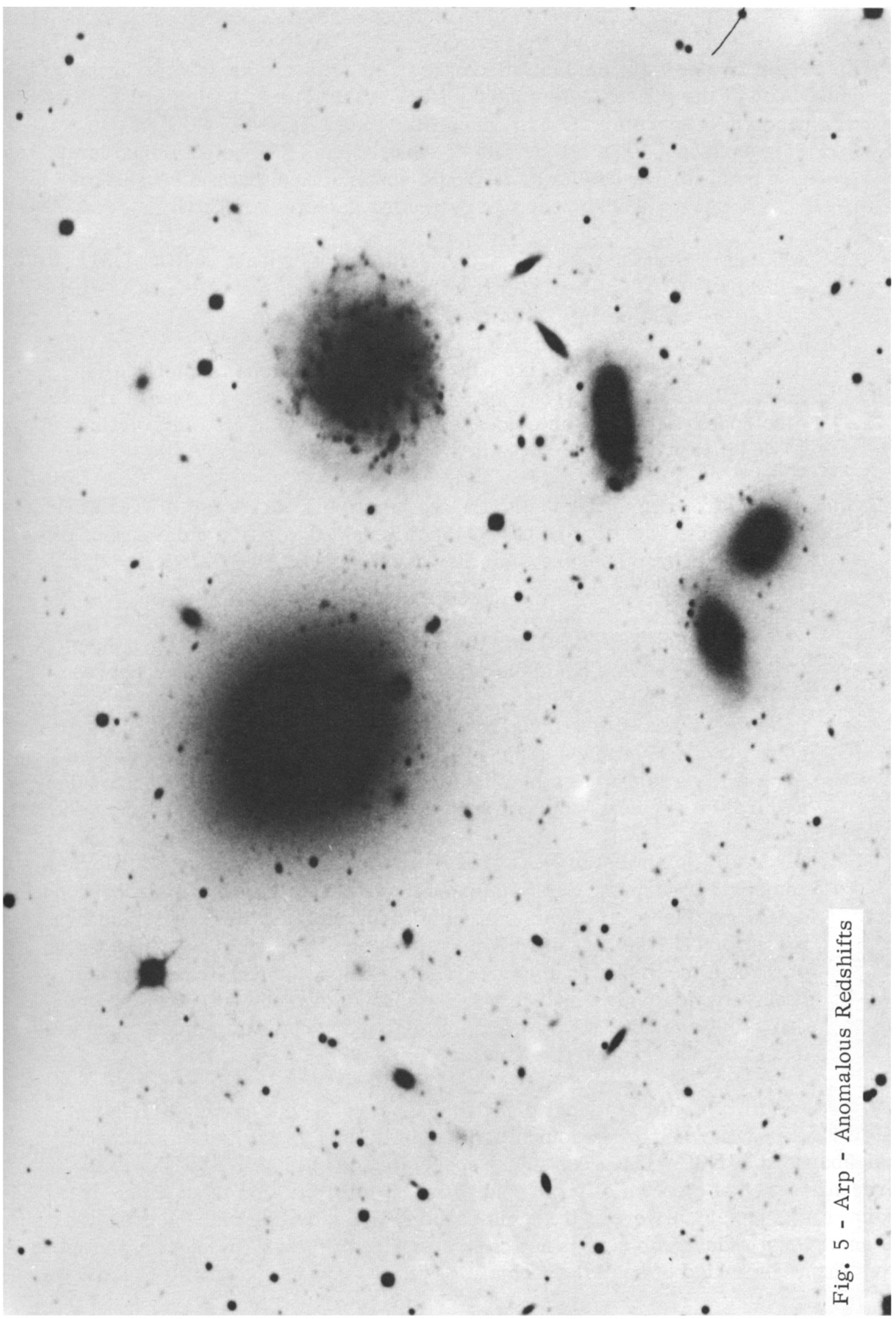


normally be expected at its true distance.

The last five new examples of discordant redshifts are all located in the neighborhood of the active Seyfert galaxy NGC 4151. The following are excerpts from a paper in preparation by Arp concerning the galaxy NGC 4151 and its surrounding environs. The full details of these objects will be published later. As a reference point for what follows, it should be remarked that the redshift of NGC 4151 is about $z=989 \mathrm{~km} / \mathrm{sec}$ (corrected for galactic rotation).

3. NGC 4156 is an Sc I spiral galaxy just to the northeast of NGC 4151 . Its redshift is $6700 \mathrm{~km} / \mathrm{sec}$, but it falls on the end of one of the NGC 4151 spiral arms and seems to be interacting with that arm.

4. There is a smaller E galaxy with faint outer envelope diametrically opposite NGC 4156 that has about the same $z=6400 \mathrm{~km} / \mathrm{sec}$. There also appears to be some evidence that this galaxy is also interacting with NGC 4151.

5. There is a straight chain of galaxies just to the northwest of NGC 4151 which points back toward NGC 4156. Redshifts for five members of the chain have been measured and they are $z=0.061, z=0.160, z=0.161$, $z=0.057$, and $z=0.160$.

6. To the north of NGC 4151 and linked to it in a common hydrogen envelope (R. Davies, private communication) is the galaxy NGC 4145. Between NGC 4145 and NGC 4151 is a dwarfish-appearing galaxy (A1208+40, also called MCG7-25-46) with narrow emission lines at $z=1045 \mathrm{~km} / \mathrm{sec}$. (Vorchkadze 1974 gives $1886 \mathrm{~km} / \mathrm{sec}$, corrected for galactic rotation.) Apparently attached to a luminous filament from this low-redshift galaxy is a spiral of luminosity class I appearance which has a redshift of $z=29,340$.

7. About $10^{\prime}$ northwest of NGC 4151 is an object designated nonequilibrium number 3 by Arp and Sulentic (unpublished investigation of neighborhoods of spiral galaxies). It consists of an absorption-line galaxy of redshift $z=36,360 \mathrm{~km} / \mathrm{sec}$ out of which emerges on a luminous filament a group of three almost stellar objects. These compact objects have emission spectra which yield a redshift of $z=58,500 \mathrm{~km} / \mathrm{sec}$. Figure 6 shows that this is obviously a case of smaller, more compact objects connected to a much lower redshift galaxy.

In general, I would comment that there appears to be a particularly large number of peculiar and anomalously high-redshift objects in the immediate neighborhood of NGC 4151. One might speculate that this active galaxy is the present source of ejection of young and peculiar objects. For the zeroeth order point of this paper, however, it seems sufficient to establish that the concentration of anomalous redshift objects is not characteristic of the sky in general and must represent some kind of spatial association with the unusual galaxy NGC 4151 . 


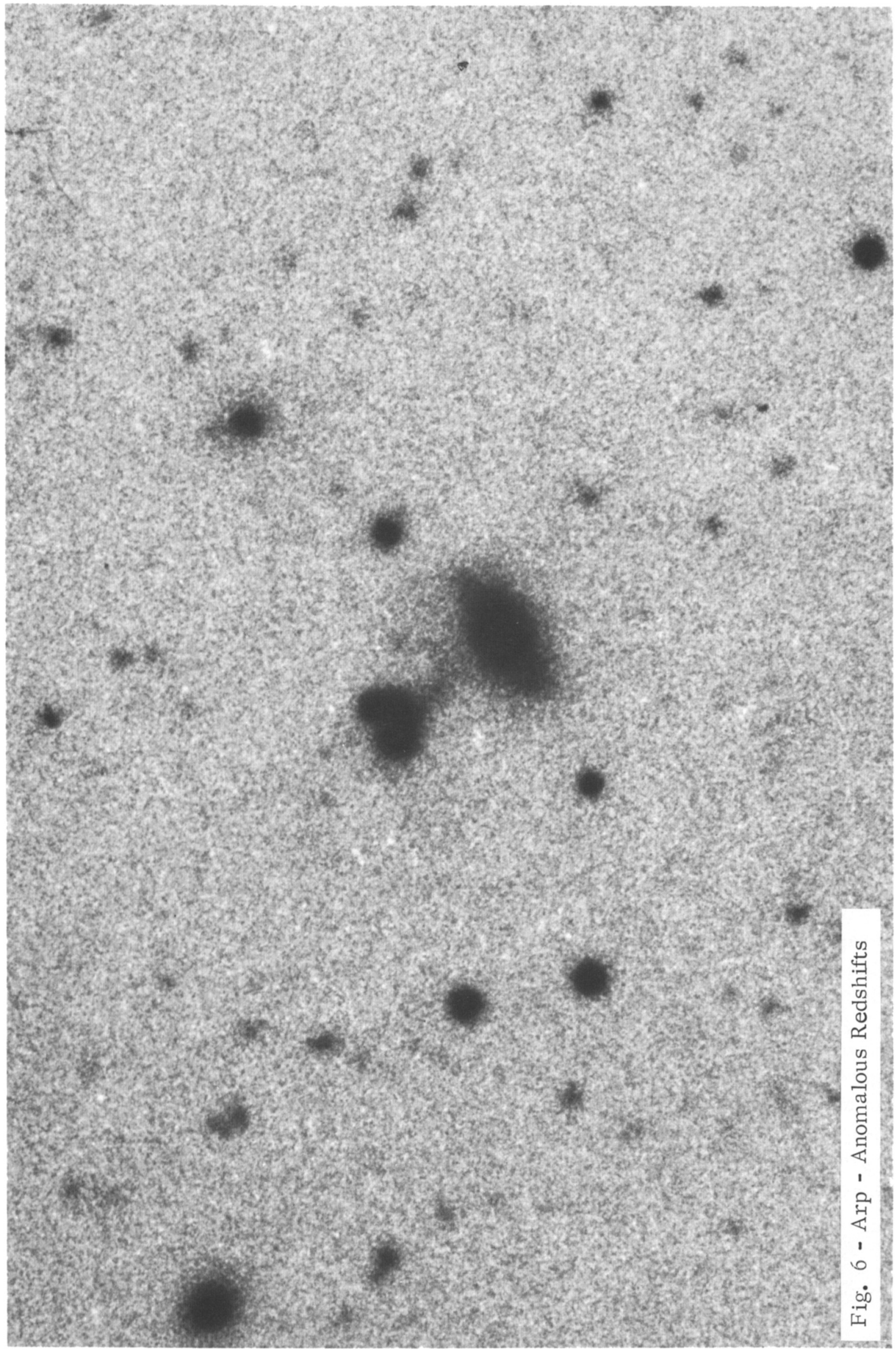


It is only when there are enough galaxies that are undeniably all together at the same distance, such as in the cases of known clusters of galaxies, that we can establish existence of intrinsic redshifts in a statistical sense. Therefore, two of the richest, the Coma cluster of galaxies and the Virgo cluster, have been considered for anomalous redshift effects (Arp 1974a).

In Coma it has been shown that the nonelliptical galaxies have mean redshifts about $+700 \mathrm{~km} / \mathrm{sec}$ greater than the ellipticals (Tifft 1972). More recently, Tifft and Tarenghi (1975) also showed that the detected radio emitting galaxies in Coma have higher redshifts than the average. In a recent analysis of the Virgo cluster, Sulentic (1976) has shown that the radio galaxies in Virgo are likewise at a higher redshift than the average of even spirals in Virgo. Jaakkola $(1971,1973)$ showed that for groups and clusters of galaxies in general, when spirals and ellipticals were at the same distance, the spirals had systematically higher redshifts. The only place where this result has been questioned is in Virgo, where there has accumulated a series of contradictory papers. The most recent (Sandage and Tammann 1976), claims there is no average difference between the spiral and elliptical members of Virgo. Sandage and Tammann do not publish the list of redshifts, including new ones of theirs, which they say vitiates the previous results. But Sulentic (1976), working with those redshifts which are published, shows that five galaxies in the Virgo direction have abruptly larger apparent diameters and lower redshifts and are, on conventional criteria, foreground galaxies. Excluding these, Sulentic gets very significantly higher redshift for the spirals in Virgo. Even including the supposed foreground galaxies, the spirals are still on the average of considerably higher redshift. One suggestion is that in the spirals, the group that Sandage and Tammann lowered in mean redshift with the addition of their unreported measures, some very faint low-redshift foreground dwarfs were added.

But there appears to be a way to answer this vexing question without leaving it forever open ended to the addition of measures on new and fainter galaxies. I would suggest that the addition of smaller and smaller galaxies and parts of galaxies do not have much physical significance for what the bulk of matter in the Virgo cluster is doing. The question which seems most meaningful is: What is the average redshift of mass contained in galaxies of elliptical morphology compared to the average redshift of the mass tied up in galaxies of spiral morphology? This question can be answered easily and definitively by weighting the redshift of each galaxy according to its luminosity. Assuming that within each morphological type there is a constant relation between mass and luminosity enables us to refer to Figure 7 and say that the material in spirals has about $600 \mathrm{~km} / \mathrm{sec}$ greater redshift than the material in ellipticals. As Figure 7 demonstrates, the mean redshifts asymptotically approach these values, and adding additional faint apparentmagnitude galaxies is not going to alter the result. 


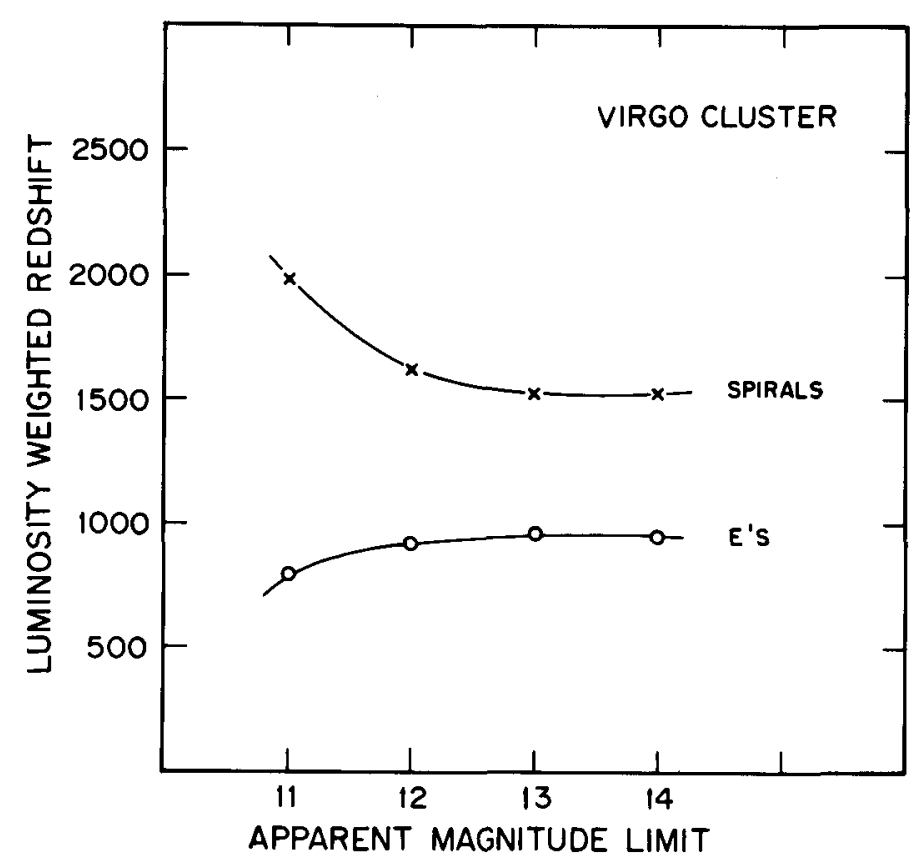

Figure 7. Published redshifts for ellipticals and spiral galaxies in Virgo cluster. Mean redshift is calculated for each morphological class by weighting according to the luminosity of each galaxy. Fainter galaxies have small effect and average weighted mean converges to very stable value for each class. (Calculations courtesy J. W. Sulentic.)

If we include the negative redshift galaxies that lie in the direction of the cluster, as we should do in what I consider a probable expanding model for the Virgo cluster, then the luminosity weighted mean difference between spirals and ellipticals is reduced to $400 \mathrm{~km} / \mathrm{sec}$. This is still large and significant, however, and it is of interest to list three new clusters discussed by J. A. Dawe, R. J. Dickens, C. Moss and B.R. Peterson at the Grenoble I. A.U. (1976, unpublished).

Table 3 lists the spiral (nonelliptical for Coma) versus the elliptical redshift differences in five clusters.

Table 3. Spiral vs. Elliptical Mean Redshifts

\begin{tabular}{lll}
\hline \multicolumn{1}{c}{ Cluster } & $\Delta z(\mathrm{~S}-\mathrm{E})$ & \multicolumn{1}{c}{ Reference } \\
\hline Coma Cluster & $+700 \mathrm{~km} / \mathrm{sec}$ & Tifft $(1972)$ \\
Virgo Cluster & +400 & Sulentic $(1976$, lum. wtd. mean) \\
A 1367 & +200 & J. A. Dawe (1976, unpublished) \\
A 262 & +140 & J. A. Dawe (1976, unpublished) \\
Cen Cl & +90 & J. A. Dawe (1976, unpublished) \\
& & \\
\hline
\end{tabular}


Of course, an investigation of the redshifts available in clusters in 1971 (Jaakkola) indicated an excess redshift of the $\mathrm{Sb}$ and $\mathrm{Sc}$ spirals over the ellipticals. It would seem justified to ask - as H. Rood did at the 1976 I. A.U. - is there a cluster known where the spirals have a smaller mean redshift?

\section{SMALL SYSTEMATIC REDSHIFT OF NORMAL COMPANION GALAXIES}

In an important paper justified by extensive lists of individual redshifts, Bottinelli and Gougenheim (1973) concluded that for a large sample of nearby groups of galaxies defined by de Vaucouleurs the mean value of redshift difference between companions and main galaxies was significant and equal to about $\Delta V=+90 \mathrm{~km} / \mathrm{sec}$. Harrison (1974) reported that he had redone this analysis and found no significant systematic effect. Nowhere in Harrison's paper, however, did it say specifically what values or procedures he disagreed with. In a matter of this importance it would seem necessary to give an item by item designation of where the differences arise. In the absence of such an account we must reluctantly consider the more detailed exposition the more reliable one.

Because it is of such fundamental importance, however, we will here look further into the matter with respect to just two of the nearest groups of galaxies about which we know the most, the M31 (Local) group and the M81 group.

Table 2 first lists the five certain companions of M31 that have been known and accepted for so long, the second column lists the redshifts that were adopted by Bottinelli and Gougenheim; and the third column lists the new redshifts and redshifts improved in accuracy that are now available. As explained in the notes to Table 2, a number of the new redshifts of Local Group members are due to Holland Ford and his co-workers at Lick Observatory. The fourth column of Table 2 lists the change in $\Delta V$ from the Bottinelli and Gougenheim values to the present values. It is seen that in every case the new values have changed so as to add to the significance of the positive residual redshifts of the companions. Of course, the newest redshift for NGC 147, which was not available previously, is the largest positive residual of all.

It is also clear from Table 2 and the plot of these values in Figure 8 that the measuring uncertainties for these redshift values are now insignificant compared to their systematic positive differences from the central galaxy.

Table 2 also lists, underneath the certain companions to $M 31$, other possible companions in order of the author's estimation of their probable association. From our own Milky Way Galaxy, M31 appears at a redshift of $V_{0}=-68 \mathrm{~km} / \mathrm{sec}$. If, as a companion to M31, our own Milky Way has an intrinsic redshift, however, when looked at from $M 31$, it would have a redshift of $\Delta V=+68 \mathrm{~km} / \mathrm{sec}$ and would plot in with the rest of M31 companions as indicated in Figure 8. As Gunn (1974) points out, the mass of M31 is not large enough to explain the fact that we should be falling toward it with $-68 \mathrm{~km} / \mathrm{sec}$. If our Galaxy is regarded as just another companion of M31, like M33 for example, then the $\Delta V$ is similar in magnitude and sign to the rest of the M31 companions. 


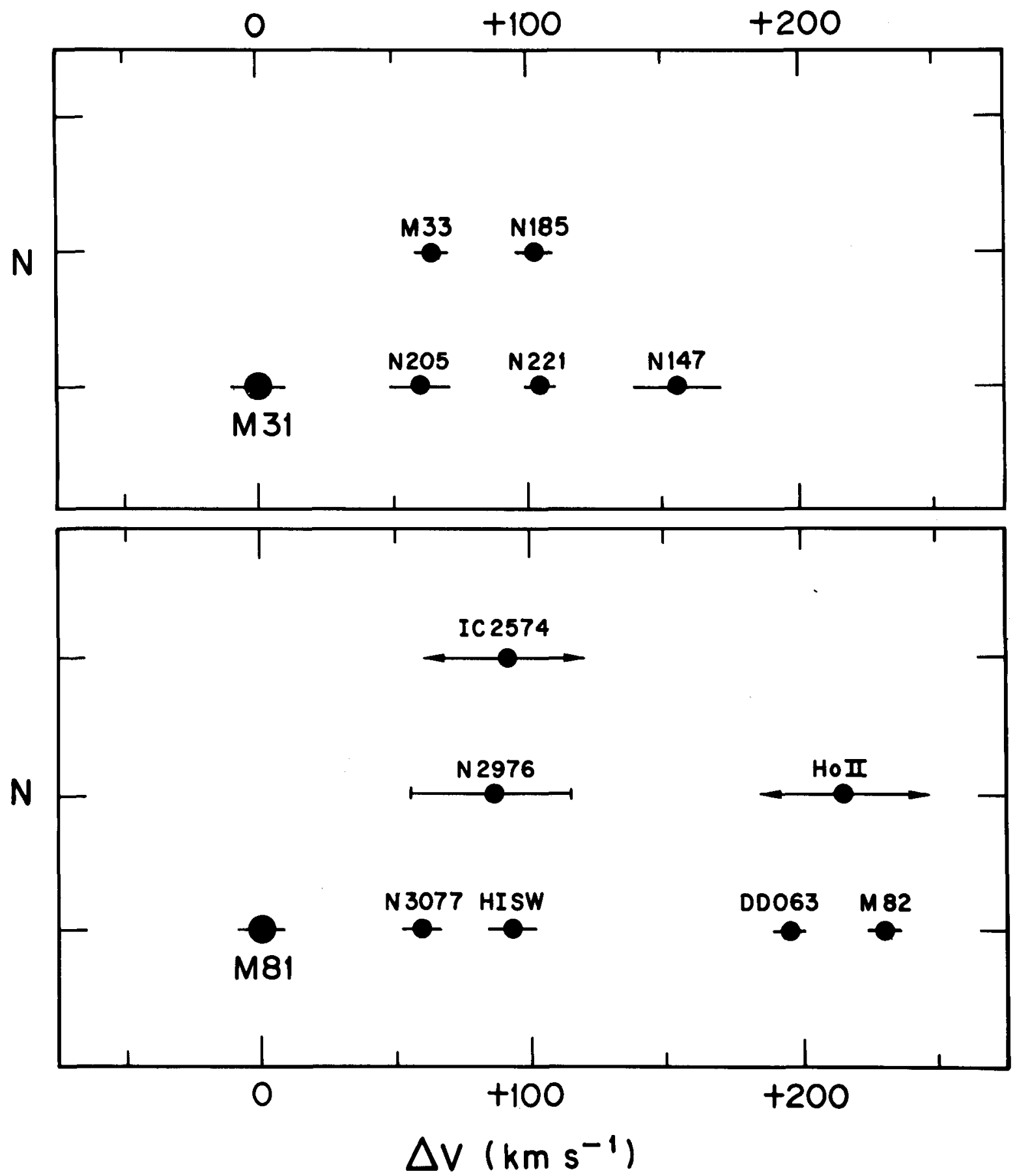

Figure 8. Histograms of residual redshifts of companion galaxies relative to central galaxies. Only companions which are certain are plotted. See Table 2. 
TABLE 2

\section{Companion Galaxies (Certain)}

\begin{tabular}{|c|c|c|c|c|c|c|}
\hline Main Gal. & Comp. & Old V $(\mathrm{km} / \mathrm{sec})$ & New V $(\mathrm{km} / \mathrm{sec})$ & $\underline{\Delta V}$ & $\begin{array}{c}\text { Change } \\
\text { (new-old) }\end{array}$ & $\begin{array}{l}\text { Notes } \\
\text { (1) }\end{array}$ \\
\hline \multirow[t]{15}{*}{ M31 } & & $-299 \pm 30$ & $-299 \pm 10$ & & & (2) \\
\hline & M33 & $-186 \pm 4$ & $-180 \pm 1$ & +63 & +6 & (3) \\
\hline & NGC 221 & $-213 \pm 10$ & $-195 \pm 5$ & +103 & +18 & (4) \\
\hline & NGC 205 & $-239 \pm 12$ & ..... & +62 & & \\
\hline & NGC 185 & $-252 \pm 30$ & $-208 \pm 4$ & +102 & +44 & (5) \\
\hline & NGC 147 & $\ldots \ldots$ & $-157 \pm 15$ & +155 & & (5) \\
\hline & \multicolumn{6}{|c|}{ Less Certain } \\
\hline & & Old $V_{0}(\mathrm{~km} / \mathrm{sec})$ & New $v_{0}(\mathrm{~km} / \mathrm{sec})$ & & & (6) \\
\hline & Milky Way & -68 & & +68 & & (7) \\
\hline & NGC 404 & +169 & & +237 & & (7) \\
\hline & NGC 6946 & +371 & & +439 & & (7) \\
\hline & IC 10 & -92 & & -24 & & (7) \\
\hline & M31 H Comp & ...... & $-220 \pm 7$ & -152 & & (8) \\
\hline & \multicolumn{6}{|c|}{ Certaln } \\
\hline & & Old $v_{0}(\mathrm{~km} / \mathrm{sec})$ & New $v_{0}(\mathrm{~km} / \mathrm{sec})$ & & & (6) \\
\hline \multirow[t]{8}{*}{ M81 } & & $88 \pm 7$ & & & & (7) $(9$ \\
\hline & M82 & $322 \pm 5$ & & +234 & & (10) \\
\hline & NGC 3077 & -26 & $+147 \pm 7$ & +59 & +173 & (8) \\
\hline & HI Comp (SW) & ...... & $+183 \pm 7$ & +95 & & (8) \\
\hline & NCC 2976 & 169 & & +81 & & (7) \\
\hline & IC 2574 & 179 & & +91 & & (7) \\
\hline & & 303 & & +215 & & (7) \\
\hline & \multicolumn{2}{|c|}{ AO $936+71$ (DDO 63) } & $+139 \pm 7(\mathrm{~V})$ & +197 & & (II) \\
\hline
\end{tabular}

Less Certain

$\begin{array}{lllll}\text { NGC } 2403 & 255 & & +167 & \text { (7) } \\ \text { NGC } 4236 & 163 & & +75 & \text { (7) } \\ \text { NGC } 2366 & & +107 \pm 7 \text { (V) } & +189 & \text { (11) }\end{array}$

(1) Old V are redshifts relative to the sun from Bottinelli and Cougenheim (1973). Their $\Delta \mathrm{V}$ had some small errors due to not correcting for galactic rotation component which have been rectified here. (2) From Rubin, V. C., and Ford, K. W., 1971, Ap. J. 170, 34. (3) New value from Warner, P. J., Wright, M. C. H., and Baldwin, J. E. 1973, M. N. 163, 163. (4) New value Ford, H. , and Jenner in preparation. (5) New value

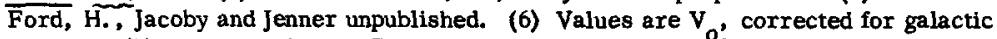
rotation. (7) de Vaucouleurs, G. and de Vaucouleurs, A. 1964, Reference Catalogue of Bright Galaxies, Univ. of Texas Press, Austin. (8) New value from Davies (1974). (9) Roberts, M. S. 1972 (ed.), D. S. Evans, "External Galaxies and Quasi Stellar Objects," I.A.U. Symposium 44. (10) Weliachew, L., 1974, Ap. J. 191, 639, and Heckathorn, H. M. 1972, Ap. J. 173, 501. (11) Material kindly supplied me in advance of publication by $\mathrm{G}$. de Vaucouleurs from 2nd Reference Catalogue of Galaxies. 
Second in the list of less certain companions of M31 in Table 2 is NGC 404 (see van den Bergh 1968). This peculiar galaxy is located on a line between M31 and M33 and has long been considered a probable Local Group member. Less likely as a candidate for the Local Group is NGC 6946. But de Vaucouleurs (1975) notes that out of the 60 nearest galaxies only 8 are unassigned to groups. NGC 404 and 6946 are two of these, so there is a slight additional reason for considering them as possible members of the Local Group. IC 10, a small, young dwarf, has been shown to be about 2.9 $\pm 1.5 \mathrm{Mpc}$ away in the Maffei group (Bottinelli, Gougenheim, and Heideman 1972), and so is probably not a member of the Local Group. Finally, there are some hydrogen clouds reported by Davies (1974), but there is a possibility that there could be some confusion with the Magellanic Stream. In summary, if the two next most probable companions of M31, which the author considers very probable, were to be plotted in Figure 8 we would then have a total of seven positive redshift residuals out of seven.

Table 2 next lists the well-known companions around M81 (de Vaucouleurs 1975). The major changes in this list since Bottinelli and Gougenheim are represented by the new hydrogen redshifts reported by Davies (1974). His value for NGC 3077 changes that differential redshift from its previous negative optical redshift with respect to $\mathrm{M} 81$ to a positive value. Davies' quoted velocity "resolution" is adopted as his measuring error in Table 2. He also reports the detection of a hydrogen companion slightly southwest of $\mathrm{M} 81$ with a positive relative redshift. The bottom half of Figure 7 plots these six certain companions of $\mathrm{M} 81$, and the last section of Table 2 lists 3 more probable companions of M81.

The net result of considering the redshifts of the companions of M31 and M81 is that 12 out of 12 of the certain companions show significant positive residual redshifts with respect to the central galaxy. If we accept probable companions, we get 14 out of 14 , and, if we accept all candidate companions, the probability calculation is at least as signiricant, 16 out of 18 . The chances of such a result occurring by accident from a population of mixed positive and negative residual redshifts is, of course, negligible.

What can the explanation be? Conventional interpretation would say that all these companions were receding from their central galaxy just along our line of sight. That is clearly untenable. Harrison (1974) pointed out that companions expanding from their central galaxies, if they got far enough in projected distance on the sky, would convert something of this expansion velocity into a positive residual from our line of sight. (Approaching the case where we are at the center of an expanding group.) But the companions in Figure 1 are mostly very close to their central galaxy and there are no evidences of any expansions in our half of the possible directions (negative residuals).

Therefore, I must conclude that there is an intrinsic component of redshift to these companion galaxies. The only suggestion that is even slightly plausible, in my view, is that these companions were formed at a later epoch than the central galaxy and that this younger matter has a higher intrinsic redshift. 
As a passing comment, we should note that Tifft (1976) has claimed that matter can occur in different redshift states and that these states are quantized in units of 70 to $75 \mathrm{~km} / \mathrm{sec}$. The distribution of residual redshifts in Figure 7 does not strongly point to a preference for such values. On the other hand it does not rule out the occurrence of such preferred values. It should be remembered, of course, that there is undoubtedly some orbital motion, plus and minus, included in those residual redshifts, and if there is ejection from the central galaxy the mean redshift of the pair must be considered. For example, if M82 and NGC 3077 were ejected exactly opposite from M81, the mean redshift of their matter with respect to M81 would be $\Delta V=\frac{+230+57}{2}=+145 \mathrm{~km} / \mathrm{sec}$.

\section{CONCLUSION}

If we accept the result that spiral galaxies have intrinsically higher redshift than ellipticals, what does this result mean in the context of anomalous redshifts of such different kinds of objects as quasars and companion galaxies? There seems to me to be only two types of unifying frameworks in which to think of these observations. One is that we have an age sequence going from quasars, as the youngest, to active and spiral galaxies, then to more related companion galaxies, and finally to elliptical galaxies as the oldest. The amount of intrinsic redshift would then decrease as the objects became older (Arp 1973). Figure 9 summarizes the intrinsic redshift as a function of morphological type in this interpretation.

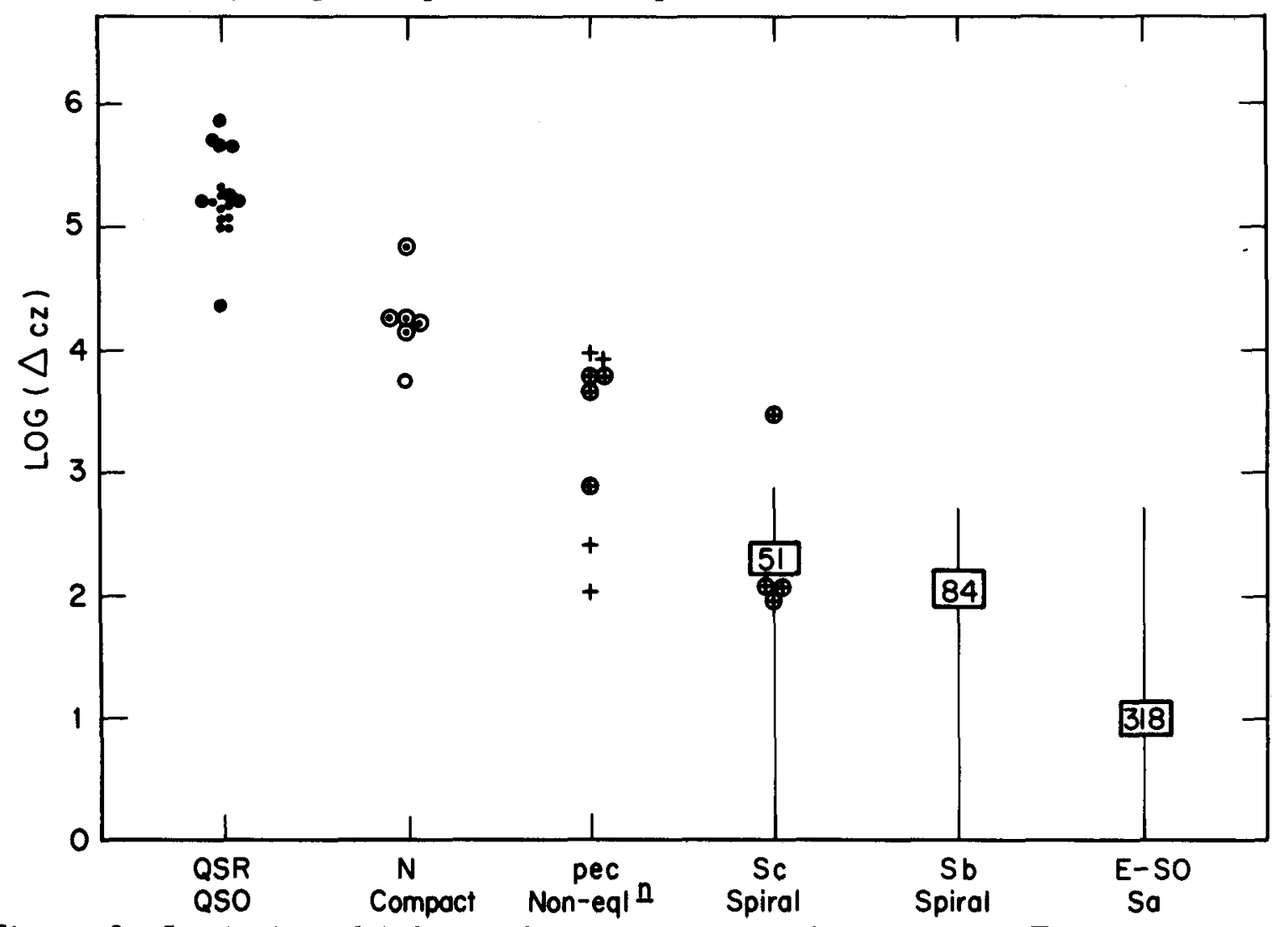

Figure 9. Intrinsic redshift as a function of morphological type. Figure summarizes data from associations of real objects, numbers in boxes refer to size of Sample in Jaakkola analysis. 
The other kind of explanation could lie in the direction indicated by Pecker et al. (1973) where the intrinsic redshift is stronger with objects more recently active in photo emission. Of course, it will be difficult to distinguish clearly at this stage the effects of mean ages from mean temperatures of galaxies. Hopefully, at the end of this colloquium those who believe the evidence is convincing enough to demand an explanation can further discuss and analyze the observational data with a view to deciding between these two broadly different theoretical interpretations.

\section{REFERENCES}

Arp, H.: 1972a, in D. S. Evans (ed.), 'External Galaxies and Quasi Stellar Objects, IAU Symp. 44, 380 .

Arp, H.: 1973, in G. B. Field (ed.), The Redshift Controversy, W. A. Benjamin, Inc., Reading, Mass.

Arp, H.: 1974a in J. R. Shakeshaft (ed.), 'The Formation and Dynamics of Galaxies, ' IAU Symp. 58, 199.

Arp, H.: 1974b in M. S. Longair (ed.), 'Confrontation of Cosmological Theories With Observational Data,' IAU Symp. 63, 61.

Arp, H.: 1976, A strophws. J., 210, No. 2 (December 1).

Arp, H. , Baldwin, J. A. and Wampler, E. J.: 1975, A strophys, J., 198, L3.

Arp, H., Pratt, N., and Sulentic, J. W.: 1975, A strophys J., 199, 565.

Arp, H., Sulentic, J. W., Willis, A. G. and de Ruiter, H. R.: 1976, Astrophys. J., 207, L13.

Arp, H., Willis, A. G. and de Ruiter, H. R. : 1975, IAU Ctrcular 2750, 25 Feb. 1975.

Browne, I. W. A. and McEwan, N. J.: 1973, MonthIy Notices Raj. Astron Soc., 162, $21 \mathrm{P}$.

Burbidge, G. R., O'Dell, S. L. and Strittmatter, P. A.: 1972, Astrqphzs. J., 175,601 .

Bottinelli, L. and Gougenheim, L.: 1973, Astron. Astrophys. 26, 85. 
REFERENCES (Continued)

Davies, R. D.: 1974 in J. R. Shakeshaft (ed.), 'The Formation and Dynamics of Galaxies,' IAU Symp. 58, 119.

Dawe, J.A., Dickens, R. J., Moss, C. and Peterson, B. R., Grenoble I. A. U. 1976, unpublished.

de Vaucouleurs, G.: 1975, (ed. Sandage, Sandage and Kristian) Stars and Stellar Systems, Vol. IX, 557.

Ekers, R. D., Fanti, R., Lari, C., and Ulrich, M. H.: 1975, Nature 258, 584.

Fanti, C., Fanti, R., Ficarra, A., Formiggini, L., Giovanni, G., Lari, C. and Padrielli, L.: 1975, Astron. Astrophys. Suppl. 19, 143

Gunn, J.: 1974, Comments Astrophys. \& Space Phys., 6, p. 7.

Harrison, E. R.: 1974, Astrophys. J. 195, L61.

Jaakkola, T.: 1971, Nature, 234, 534.

Jaakkola, T.: 1973, Astron. Astrophys. 27, 449.

McEwan, N. J., and Browne, I. W. A.: 1975, Mem. Roy. Astron. Soc. 80, 1.

Pecker, J. C., Roberts, A., Tait, W. and Vigier, J. P.: 1973, Nature 241, 338.

Sandage, A. and Tammann, G. A.: 1976, Astrophys. J. L1.

Strittmatter, R. A., Carswell, R. F., Gilbert, G. and Burbidge, E. M.: 1974, Astrophys. J. 190, 509.

Sulentic, J. W.: 1976, Astrophys. J. submitted.

Tifft, W. G.: 1972, Astrophys. J. 175, 613.

Tifft, W. G.: 1976, Astrophys. J. 206, 38.

Tifft, W. G. and Tarenghi, M.: 1975, Astrophys. J. 199, 10.

van den Bergh, S.: 1968, Communtcation David Dunlap Obs. No. 195.

Vorchkadze, T. M.: 1974, Astrofizika 10, 493.

Weedman, D. W.: 1971, Astrophys. Letters 9, 49.

Wills, B. J., Wills, D. and Douglas, J. N.: 1973, Astron. J. 78, 521. 


\section{DISCUSSION}

T.K. MENON: We know that phenomena with different scales occur in radio quasars. However the largest scale phenomena are represented by the double radio sources. Accepting the cosmological interpretation of quasar redshifts the distribution of linear size against radio luminosity for double quasars has a strong peak at around 80 to $100 \mathrm{kpc}$, indicating that these double sources reach their maximum luminosity when dimensions reach galactic dimensions. I cannot believe that this is a coincidence. I suggest that this aspect of the quasar phenomena provides a strong support for the cosmological interpretation of quasar redshifts.

H. ARP: This brings up the point that Petrosian tried to make and which Bahcall also misunderstands. My local model, as described in the literature, has the quasars mostly in the Local Group of galaxies and the high redshift ones of lower intrinsic luminosity. This is what the observational evidence indicates to me. Naturally this leads to a situation where we would observe the highest redshift quasars at the faintest apparent magnitudes (and probably physically smaller). So it is not true as Menon implies, and as Petrosian states, that the observed average $\mathrm{z}, \mathrm{m}$ for quasars rules out the local model at greater than the $99 \%$ confidence level. In fact I am rather annoyed that he would make this statement when the precepts of the local model are clearly stated in the literature.

J.P. VIGIER: a) There is apparently now strong evidence for two sets of QSOs since Maarten Schmidt has shown that there is no sign of evolution for flat QSOs.

b) The only possible "memory" would be the rotation axis at the mother object. Could someone try to check if this axis fits with the orientation of some chains?

c) Maybe the NGC 199 can be turned into a crucial test by an analysis with the electronic camara?

V. RUBIN: Perhaps Dr. Arp has neglected two other objects in the M 31 tabulation. During his observations of M 31, Baade discovered 2 small galaxies between the arms, with $\mathrm{V} \sim 16,000$ and $30,000 \mathrm{~km} \mathrm{~s}^{-1}$. They are strong emission line objects, but not at all like normal emission line galaxies at these velocities, because they appear to have no visible outer struc- 
ture on copies of his 100" plates. Moreover to the best of my recollection, at least one of them appears very near the larger emission complex at 96' studied by Baade \& Swope, and thus not really "between the arms".

Some years ago I obtained copies of Baade's plates and found a note written by Baade to Mayall apparently the morning after Mayall had obtained a plate showing the large velocity. Baade wrote "Very remarkable are the blue nebulosities a) and $b$ ). There is no indication of emission but they are so blue that one would expect spectra of type A - F or maybe earlier. They can hardly be extra-galactic and appear to belong to $\mathrm{M} 31$. Perhaps one could get a spectrum of a) which would be useful for radial velocity. Was not b) the object for which you obtained last year $R V=+27200 \mathrm{~km} \mathrm{~s}^{-1}$ ? If that's the case I am more thrilled than ever because the large redshift and the intrinsic "blueness" are an extraordinary combination. Maybe we have stumbled on a gold nugget! Next day: The nebula in question is obviously an extragalactic system in which early type stars predominate. With increasing red shift such a system should become bluer and bluer and photographically brighter and brighter. Probably Hubble will not like that because it indicates that his random corrections for redshift cannot be handled that way. In any event it is a nice case to remember." (Finding charts for $a$ and $b$ are located in Humason, Mayall, and Sandage, Astron. J. 61, plate facing p. 128. - Spectra of b) was dated Sept. 1946. - Date of note might be 1947 ).

E. KHACHIKIAN: I would like to show two examples, where the galaxy is
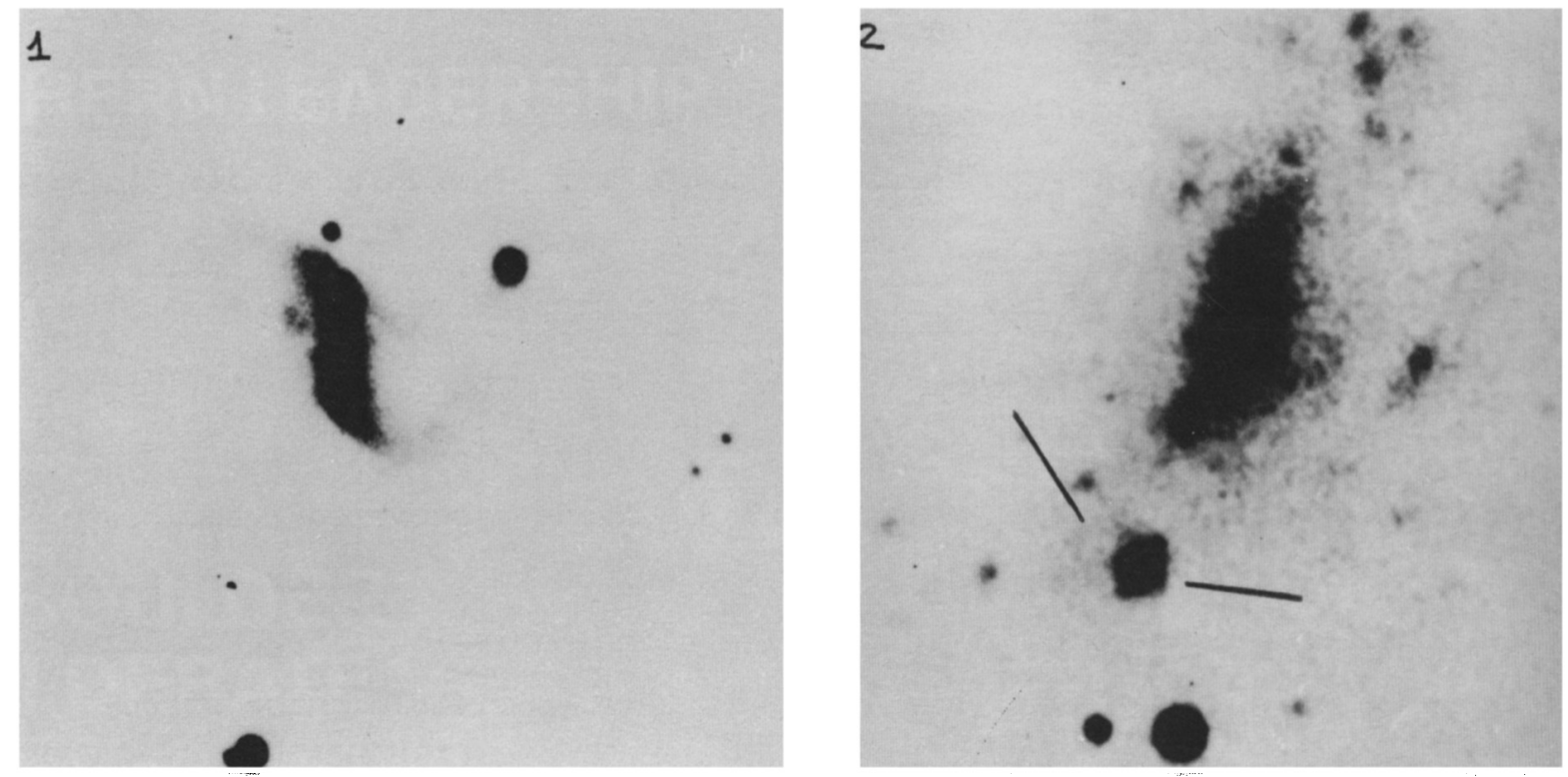
connected with the compact object and both have the same redshift.

First slide shows Markarian 305 (compact) and Markarian 306. Markarian 306 is a spiral galaxy with a complicated, and maybe destroyed nucleus, and with strong emission line spectrum, whereas Markarian 305 is very compact with only absorption lines. The photograph is obtained with the new $6 \mathrm{~m}$ telescope of Special Astrophysical Observatory of Academy of Sciences of USSR. (Original Scale: $1 \mathrm{~mm} \sim 8 "$ ).

Second slide shows Markarian 94 (compact) and III Zw $0843+51$. Actually Markarian 94 is a superassociation in the galaxy. Between Markarian 94 and the central part of the galaxy there is another compact object, which is also a superassociation. All three: Markarian 94, the compact object, and the nucleus of the galaxy have the same redshift. The photograph was obtained by Arp with the 200" telescope.

J.-C. PECKER: The $\Delta \mathrm{v}$ in groups of galaxies, as studied by Arp, or Bottinelli - Gouguenheim, has also been looked at by Collin-Souffrin, Tovmassian, and myself (Astron. Astrophys. 30, 351, 1974). By measuring the Byurakan type (which measures both the compactness, and the UV excess) we found that the bluer the object is the bigger is the redshift. The evidence was not very convincing, but together with other evidence, such as described by Vera Rubin a minute ago, we are led to associate the anomalous redshift and the nature of the redshifted object.

S.M. FABER: A systematic effect which might act to cause the difference in redshift between spirals and ellipticals in clusters is due to possible background contamination of the spiral population, which is much less concentrated to the cluster center. It is interesting that Virgo shows this effect rather strongly and Abell's recent work on Virgo demonstrates that the density of background groups in Virgo is rather high. His classification of spirals in the Coma region also suggests that there may in fact be few if any true spiral members of the Coma cluster. The question is clearly very complicated, and it requires a very careful consideration of the membership issue.

M. ROWAN-ROBINSON: Naturally I support Dr. Vigier's remark about the importance of the different rates of evolution in flat-spectrum compact quasars and steep-spectrum extended quasars (cf. Nature 1972, 236, 112). 
The absence of evolution in the compact quasars could indeed be because they are a local population, although I personally do not believe this now. An alternative explanation, on the cosmological hypothesis, is that we are looking at the generator of the whole active galaxy phenomenon (extended sources, optical non-thermal cores, etc.) and that this merely reflects the rather slow evolution expected in the nucleus of a normal galaxy of gas and stars.

Turning to the question of quasars near bright galaxies, I do not think there is much problem in understanding why there is a correlation of QSO separation with galaxy diameter or redshift. Looking at Dr. Arp's new data, most of the QSOs reported as associated with bright galaxies are within about 3 Holmberg diameters of the galaxy, which suggests that this is the size of the typical region searched. QSOs at larger separations would not be considered "of interest". The lower envelope would be defined by about $0.5-1$ Holmberg radius.

The crucial question is the one of surface density of QSOS. Looking at Arp's data as a whole, and assuming that 3 Holmberg diameters have been searched around each of these galaxies (plus the fact that about a further 10 galaxies have been searched unsuccessfully - Arp, private communication), the surface density of optical QSOs does not seem to be significantly higher than that given by Braccesi and Formiggini, or Sandage and Luyten.

This suggests a crucial test: the vicinity of these Arp galaxies should be searched for QSOS in a systematic way and the results compared with a similar number of random fields.

W.G. TIFFT: The elliptical-spirals redshift difference in Coma quoted by Arp refers to the $E / S O$ difference in the very central regions. Background can play no significant part. A second sample of more outlying objects is the emission line galaxies, clearly not ellipticals and equally clearly a part of the cluster. They also show a redshift excess over the cluster mean or elliptical galaxy mean. In the $\mathrm{E} / \mathrm{SO}$ case, cloud motions might be invoked to explain some of the difference. The extended symmetrical pattern of the emission line galaxies, however, is not easily explained by cloud motion.

S. VAN DEN BERGH: A recent reanalysis of radial velocities within $6^{\circ}$ of the centre of the Virgo cluster (Vistas in Astronomy, in press) shows 
that the mean velocity of spirals does not differ from that of ellipticals at a statistically significant level.

M. BURBIDGE: I would just like to add a few words on the remarkable system 3C 303, which was described yesterday by $\mathrm{Dr}$. Kronberg. The fact that more than one optical object was involved in the radio source goes back to Wyndham's work. The importance of the very unusual trio of UV-excess objects, discovered by Lelièvre and wlérick, was pointed out to me by Dr. Veron - radio work in France had shown that the radio structure involved them. Some years elapsed before the Wampler-Robinson scanner at Lick made it possible to measure the brightest of the UV excess objects. In the meanwhile, new radio data had been obtained by Kronberg, and that together with his most recent data, make it clear that we are concerned with a single complex radio source, not a chance coincidence of independent sources. While a priori statistical arguments now exist by which one could calculate a probability for finding a QSO 18 arcsec from the $3 \mathrm{C}$ radio galaxy, it is, as $\mathrm{Dr}$. Kronberg stated, the peculiarity of both radio and optical properties (triple UV-excess objects within a few arcsec of each other and involved in the radio source) that makes it difficult to apply statistical arguments here. Regarding the angular separation of the QSO from $\mathbb{N}$-type galaxy, I do not know how this compares with the relation mentioned by Arp.

J.L. NIETO: I would like to present here a different look at the peculiar problem of the quasars-galaxies associations. We have used a complete list of quasars kindly communicated to us by Dr. Véron and the galaxies from

Comparizon of the observed and expected distributions of angular distances, for the adopted value of the mean density of galaxies.

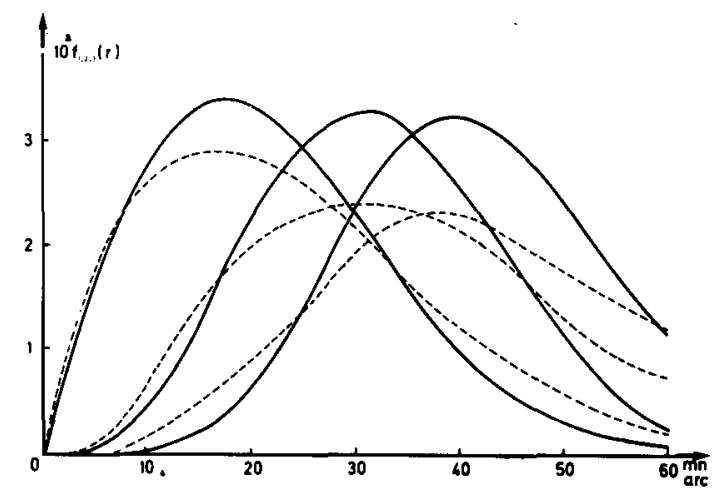


Zwicky's catalogues with magnitudes inferior to 15.7. We have established a local mean density of galaxies around each quasar and computed the angular distances between each quasar and its three nearest galaxies. We have corrected these angular distances from the corresponding densities of galaxies and adopting a mean value of 1.79 galaxies per square degree, and we have compared the three new distributions of corrected angular distances with the three expected distributions if the galaxies are randomly distributed around each quasar with the adopted value of the density. The results are shown in the figure where the three smoothed observed distributions are plotted with dashed lines compared to the three expected distributions. We notice a slight tendency for some quasars to be nearer to the galaxies than expected, but at the same time for others to be farther away than expected. That means that we have a larger dispersion for the observed distribution than for our expected distribution.

We have the same result for the angular distances distributions between $3 \mathrm{C}$ quasars and the brightest galaxies (magnitudes smaller than 14.0). Indeed, if we observe the four very close associations detected by Dr. Burbidge et al., we also observe significantly higher values of the angular distances than the expected ones for the corresponding mean local densities of galaxies. And we have again the same result for $3 \mathrm{C}$ quasars and galaxies with a magnitude inferior to 15.7 .

We have to explain this abnormal dispersion. There are probably two reasons: 1) Uncertainties in the determinations of the local mean densities of galaxies. 2) A very local clustering of galaxies around each quasar not taken into account.

Some further investigations using arbitrary random distributions are now in progress to examine whether these reasons are correct. This study suggests that the very small angular distances detected between quasars and galaxies only correspond to the foot of the distribution shown here, and are very seldom cases when we use nearly homogeneous samples of quasars.

our results are basically statistical. This means that we cannot be sure to eliminate every case of very close pairs; and there are probably not so many doubtful cases as believed. On the other hand, our result does not exclude possibilities of associations when the concerned 
galaxy magnitudes are superior to 15.7. Does it mean or not that quasars are at their so called cosmological distances? Nothing in the results of our study allow us to conclude about it.

Furthermore, to answer Drs. Vigier and Rowan-Robinson, we have divided the radioquasars in our list in the two classical classes following the slopes of the radio spectra. We notice a slight tendency for flat quasars to be nearer to the galaxies than steep quasars. This tendency is more or less important according to the different classifications (until $2 \sigma$ level), but does not reach a statistical significance when we take into account possible selection effects.

B.M. LEWIS: There are some mistakes in Bottinelli and Gouguenheim's published paper on companion galaxies. When these are rectified and all available $21 \mathrm{~cm}$ and optical observations (avajlable in 1973) are used on the same groups under the same circumstances as done by Bottinelli and Gouguenheim, and an average of $\Delta$ (companion-dominant) formed which is weighted by the associated standard errors, then $\langle\Delta \mathrm{V}\rangle=32 \pm 33 \mathrm{~km} \mathrm{~s} \mathrm{~s}^{-1}$. If instead, we use all possible de Vaucouleurs groups for which sufficient data exist and choose the dominant galaxy as the brightest (after due consideration of its type and total magnitude), and form the difference $\Delta \mathrm{V}=$ average-velocity of all companions $-\mathrm{V}$ (dominant) then the arithmetic $<\Delta \mathrm{V}\rangle=+17 \pm 43 \mathrm{~km} \mathrm{~s}^{-1}$ from 51 groups. These two differences are typical of the means formed, whatever variation is made to the inclusion or exclusion of arguable groups.

L. GOUGUENHEIM: My opinion today is that these kinds of statistical studies of de Vaucouleurs' groups strongly depend on the constitution of each group. More recent studies by Tully and Fisher have shown that many of these groups can be divided in subgroups with lower internal velocity dispersions.

It appears thus better to restrict this kind of studies to the only well-known groups for which the membership is certain, as Dr. Arp did in his talk.

J.-C. PECKER: I do not believe Ryle's argument about the non-likelyhood of local nature of QSS: the reduction of distance leads accordingly to a reduction of the energy output per unit of volume. In other terms, the "Ryle paradox", is similar to the "Olbers paradox"; hierarchical distribu- 
tion may be an answer.

T. JAAKKOLA: The following comments on the quasar problem are on a rather general level. I consider in particular the question of the existence of two populations of radio-emitting quasars, one, called $(f, c)$ quasars, with compact radio sources and flat radio spectra, belonging to the class of dwarf galaxies, and the other $(s, e)$, with extended sources and steep spectra and belonging to the class of giant galaxies. Evidence in favor of such distinction in luminosity has been published recently (Jaakkola et al. Astrophys. Space Sci., 37, 301, 1975). The (f,c)-quasars are associated with nearby clusters and the $(s, e)$ - quasars with distant clusters. Division into two luminosity populations was earlier suggested by Rowan-Robinson (Nature 236, 112, 1972; Astron. Astrophys. 23, 331, 1973) who later (Nature 262, 97, 1976) rejected this idea.

At the same time as I consider the evidence given as significant, it is still worthwhile to ask whether such a distinction of two populations of objects appearing quite similar morphologically and having some similar spectrum characteristics, is plausible or not. It is known from the very history of the discovery of quasars that apparently identical stellar-like objects, namely stars and QSOS, can be located at vastly different distances. Likewise, high surface brightness dwarf galaxies at local distances appear similar to giant galaxies with bright ruclei at larger distances, the disk of the giant galaxy shrinking there inside the bright image of the rucleus. Such high surface brightness dwarf and giant galaxies are known to exist, and in extreme forms they might be quasars. Therefore, morphologically the existence of two Iuminosity classes of quasars is quite natural.

With the exception of the optical form and some spectral features the two quasar classes are far from being similar: One, $(f, c)$ is varying, the other is not. The largest redshifts are found for the $(f, c)$-class. The polarization properties are different. In the radio band the structural and spectral properties are completely different. These dissimilarities must reflect basic differences in the nature of these objects, including their mass and luminosity.

But there are also similarities: high redshifts, wide hydrogen lines etc. This is not unexpected. Quasars of both classes, although with 
luminosity differences of the order of 100, may have their origin in a rather similar event, an explosion in a galactic nucleus. In the $(f, c)$-case an expulsion of a coherent body by a nucleus of a giant galaxy would have resulted in the quasar as a satellite of the galaxy, as seen in many associations presented by Arp. In the case of the $(\mathrm{s}, \mathrm{e})$ -quasars the explosion may have happened in a diffuse and spherically symmetric manner, creating a bright nucleus - $(s, e)$-quasar - in the galaxy itself. Both in $(f, c)$ and in $(s, e)$-quasars matter would be in a post-explosion state, and certain spectral similarities are therefore not surprising.

In short, once the possibility of the existence of non-velocity redshifts is accepted, prediction about two Iuminosity populations of quasars can be made on the basis of the morphology of high surface brightness galaxies and known non-stationary phenomena in galactic nuclei. Perhaps such a prediction could have been made even without the present strong evidence of these populations, had astronomers read the lesson given by the discovery of quasars more carefully.

J. TERRELL: Certainly one would expect to see local quasars emitted by other galaxies, in addition to our own, so that some associations of faint quasars and bright galaxies of the sort just reported by Arp would be in good agreement with these ideas. No difficulty for local quasars should arise here, or in the $21-\mathrm{cm}$ absorption lines just reported in 2 quasars by Burke, as long as the number of cases, all with redshifts, does not grow too large.

As to the remark attributed to Ryle, there seems to be no problem there for very local quasars. One would expect, on the very local basis, to find radio emission near some other galaxies (Science 154, 1281, 1966; Astrophys. J. Letters 147, 827, 1967), and this has in fact long since been found, with the discovery of radio galaxies!

B.F. BURKE: The two quasar-galaxy pairs that show hydrogen absorption in the quasar spectrum are a factor of 30 or so more distant than Dr. Terrell's local quasars. They would show 7 or 8 magnitudes brighter if similar objects were near our galaxy. This would seem to make them highly luminous objects indeed. Also, it is curious that all the quasar-galaxy pairs show the quasar receding. 
M. ROWAN-ROBINSON: In spite of my defection, I think we should continue to be fair to the "Iocal" hypothesis, which I do not think Dr. Burke is being. Some time ago (Astron. Astrophys. 23, 33, 1973) I worked out in some detail the implications for the radio source-counts and integrated background of bringing the quasars in closer than their cosmological distances. The whole population can be brought in by a factor of about 100 without contradiction. And if only some of the QSOs are "Iocal" (eg. the flat-spectrum, compact quasars), the limit is much weaker - they can be brought in by a factor of 300 or more.

P.C. VAN DER KRUIT: Using the Westerbork radio telescope Mr. van der Hulst in Groningen mapped the HI in the M 81 group and among other results found a thin "stream" of hydrogen connecting NGC 3077 directly to one of the arms of $\mathrm{M} 81$. I have two remarks in connection with the discussion. In the first place, since this question arose earlier, the distribution of HI in the M 81 group is indeed as patchy as the detection rate of absorption of 2 out of 8 , reported by Burke, would require. In the second place Arp's implication that $\mathrm{NGC} 3077$ has an intrinsic redshift relative to $M 81$ seems difficult to reconcile with these observations. Along the stream the velocity varies smoothly from that of the HI around NGC 3077 until it joins the velocity field of M 81. There should therefore be a smooth variation of intrinsic redshift along the stream, possibly even continuing in the velocity field of M 81. To my mind this renders an intrinsic redshift in NGC 3077 unacceptable.

C. HAZARD: It is true that in an identification programme of Molonglo radio sources in a $2^{\circ}$ wide declination strip centrered on $11^{\circ} \mathrm{N}, \mathrm{H}$. Murdoch and myself have found an apparent variation in QSO density with the highest density and brightest QSOs in the interval $11^{\mathrm{h}} 30^{\mathrm{m}}-12^{\mathrm{h}} 30^{\mathrm{m}}$, that is in the Virgo region. The full significance of this work must await the completion of the whole identification programme. I personally will be convinced only if the density fluctuations are correlated with differences in the redshift distributions. Some of the newer results from the Lick and Steward observatories indicate that this may indeed be the case. In the Virgo region no more than one third of the QSOS have $z>1.5$ and only one or two just over $z=2$, while in the $8^{\mathrm{h}}$ region, where the QSOs appear to be fainter and less common, the 11 so far measured all have $z>1.5$. 
The high density at $12^{\mathrm{h}}$ may have implications for some of the statistical associations, such as the correlations between bright galaxies and QSOs for it suggests that the QSO density in some regions may be higher than is generally assumed. For a radio flux $\geq 0.45$ there are about 0.5 QSOs brighter than 19.5 per square degree. The total number radio quiet objects is probably at least $5-10$ per square degree brighter than 19.5 . 